\title{
WHO malaria nucleic acid amplification test external quality assessment scheme: results of distribution programmes one to three
}

Jane A. Cunningham ${ }^{1 *}{ }^{*}$, Rebecca M. Thomson ${ }^{2 \dagger}$, Sean C. Murphy ${ }^{3}$, Maria de la Paz Ade ${ }^{4}$, Xavier C. Ding $^{5}$, Sandra Incardona ${ }^{5}$, Eric Legrand ${ }^{6}$, Naomi W. Lucchi $^{7}$, Didier Menard ${ }^{6}$, Samuel L. Nsobya ${ }^{8}$, Agatha C. Saez $^{9}$, Peter L. Chiodini ${ }^{9,10}$ and Jaya Shrivastava ${ }^{9,10}$

\begin{abstract}
Background: The World Health Organization (WHO) recommends parasite-based diagnosis of malaria. In recent years, there has been surge in the use of various kinds of nucleic-acid amplification based tests (NAATs) for detection and identification of Plasmodium spp. to support clinical care in high-resource settings and clinical and epidemiological research worldwide. However, these tests are not without challenges, including lack (or limited use) of standards and lack of reproducibility, due in part to variation in protocols amongst laboratories. Therefore, there is a need for rigorous quality control, including a robust external quality assessment (EQA) scheme targeted towards malaria NAATs. To this effect, the WHO Global Malaria Programme worked with the UK National External Quality Assessment Scheme (UK NEQAS) Parasitology and with technical experts to launch a global NAAT EQA scheme in January 2017.
\end{abstract}

Methods: Panels of NAAT EQA specimens containing five major species of human-infecting Plasmodium at various parasite concentrations and negative samples were created in lyophilized blood (LB) and dried blood spot (DBS) formats. Two distributions per year were sent, containing five LB and five DBS specimens. Samples were tested and validated by six expert referee laboratories prior to distribution. Between 37 and 45 laboratories participated in each distribution and submitted results using the online submission portal of UK NEQAS. Participants were scored based on their laboratory's stated capacity to identify Plasmodium species, and individual laboratory reports were sent which included performance comparison with anonymized peers.

Results: Analysis of the first three distributions revealed that the factors that most significantly affected performance were sample format (DBS vs LB), species and parasite density, while laboratory location and the reported methodology used (type of nucleic acid extraction, amplification, or DNA vs RNA target) did not significantly affect performance. Referee laboratories performed better than non-referee laboratories.

Conclusions: Globally, malaria NAAT assays now inform a range of clinical, epidemiological and research investigations. EQA schemes offer a way for laboratories to assess and improve their performance, which is critical to safeguarding the reliability of data and diagnoses especially in situations where various NAAT methodologies and protocols are in use.

Keywords: External quality assessment, Malaria, Molecular, Proficiency testing

*Correspondence: cunninghamj@who.int

${ }^{\dagger}$ Jane A. Cunningham and Rebecca M. Thomson joint first authors

${ }^{1}$ World Health Organization, Geneva, Switzerland

Full list of author information is available at the end of the article

\section{Background}

Malaria remains a global health challenge and despite recent advances still accounts for more than 400,000 deaths annually, with $90 \%$ of deaths occurring in 
Africa [1]. A key aspect of control and elimination of this disease is accurate diagnosis and since 2010 the World Health Organization (WHO) has recommended parasite-based diagnosis of malaria in place of syndromic treatment. Microscopy and rapid diagnostic tests (RDTs) are the main tools used for malaria case management and routine surveillance in endemic countries [2].

Over the last 30 years, important advances in molecular techniques have led nucleic acid amplification-based tests (NAATs) to be increasingly used for identification of Plasmodium infection. Abundant nucleic acid targets were first recognized in the 1980s by Waters and McCutchan [3]. Polymerase chain reaction (PCR) was first applied to Plasmodium by Snounou and colleagues in 1993 [4], which more easily enabled detection of Plasmodium nucleic acids in a blood sample. Since the advent of conventional PCR, numerous additional modifications have been developed, including nested PCR, real-time PCR, multiplex PCR, reverse transcription PCR, and loop-mediated isothermal amplifications (LAMP) [5-12].

These more sophisticated NAATs allow for species identification with use of species-specific primers or melt curve analysis, detection of mixed infections, and some NAATs can be used to estimate parasite density. NAATs have excellent sensitivity, down to $1-20$ parasites $/ \mathrm{mL}$ of whole blood in the most sensitive assays, depending on the volume of blood tested $[12,13]$, and permit earlier detection of infections compared to other diagnostic methods [9]. As NAATs can also be used to test archived material, samples can be conveniently analysed retrospectively.

NAAT have been increasingly used in epidemiological studies as well as the reference standard for malaria infection in evaluations of new diagnostic tests, in clinical trials assessing the efficacy of anti-malarial medicines and vaccines, and in clinical case management in highincome countries [14-16]. However, outside of these mostly resource-rich settings, the cost, specialist training and equipment requirements have largely restricted NAAT use to epidemiological and clinical trial research. In low-transmission endemic settings the greatest proportion of Plasmodium falciparum and Plasmodium vivax infections are at low density, below the limit of detection of microscopy and RDTs $[17,18]$, and such infections contribute to the transmission of malaria [19, 20]. In those areas, NAATs that enable the identification and treatment of such infections may accelerate elimination efforts such as screen and treat programmes and targeted malaria elimination strategies [21].

Many different NAAT methods are reported and are in use; however, there is a lack of or limited use of common standards used in different laboratories and a lack of formal external quality assessment (EQA) programmes for these methodologies [14].

Rigorous quality control is critical to ensuring that reliable and comparable results are generated by NAATs. Quality assessment activities, such as proficiency testing, can promote improvement in laboratory performance and alignment of methodology [22-25]. Amongst centres performing controlled human malaria infection (CHMI) studies, a previously reported EQA study showed high rates of agreement between centres [16]. However, beyond these highly regulated clinical trial sites, there is evidence of a lack of alignment between molecular diagnostic laboratories [26, 27], and there has not been wide uptake of international standards, such as the WHO DNA standard for $P$. falciparum $[26,28]$. Malaria NAAT EQA or 'proficiency testing' schemes are a rarity, even in high-income countries [16]. EQA programmes for other malaria diagnostic tools, such as microscopy, have resulted in improved performance of these tests [29], while independent performance evaluation schemes of RDTs have resulted in shifts in the market towards better quality products [30].

Due to the heterogeneity of NAAT methodologies in use and reliance of policy making on research using NAATs as a reference standard, the WHO Global Malaria Programme commissioned the development of a repository of EQA materials for malaria NAATs. Together with the UK National External Quality Assessment Service (UK NEQAS) as the service provider, a NAAT EQA scheme informed by an expert consultation was started in January 2017 [31]. Participation was free of charge, and results were provided confidentially to the participating laboratories.

The scheme enables individual laboratories to assess their performance over time and, where necessary, troubleshoot and make improvements in their methodology. Across all participating laboratories, where factors associated with superior performance are evident, they are likely to promote harmonization of malaria NAATs among laboratories performing these techniques. Results from the first three distributions of this EQA scheme are presented in this paper, however the scheme is ongoing. The challenges encountered are also discussed, along with how the results of the scheme have been used by participating laboratories to improve their performance.

\section{Methods \\ Enrolment of laboratories}

Between December 2014 and January 2015, a survey regarding malaria NAAT activities was circulated widely through public health and research laboratory networks to reference and research laboratories in all WHO regions. Contact details were identified through 
a range of laboratory networks supporting clinical trials of medicines and vaccines, malaria elimination research and surveillance. Subsequently, 55 survey respondents who reported that they perform NAAT for Plasmodium detection were invited to participate in the EQA scheme, consisting of two distributions per year, on condition of signing a letter of agreement with WHO. Following presentations at international conferences and development of a WHO webpage featuring documentation describing the scheme, other laboratories joined in distributions 2 and 3 and more have joined in subsequent distributions.

\section{EQA source materials}

Eighty-six per cent of $P$. falciparum and all Plasmodium knowlesi samples were sourced from in vitro cultures of laboratory strains prepared in the London School of Hygiene and Tropical Medicine (LSTMH). The other 14\% of $P$. falciparum samples, as well as samples for other species, were prepared from leftover clinical specimens from patients attending the Hospital for Tropical Diseases in London and from residual blood samples referred from other hospitals across the UK. Under the terms of the UK Human Tissue Act, 2004, Schedule 4, Section 45, supplementary part 2, ethical approval to use such samples for EQA purposes is not required [32].

\section{Preparation of EQA samples}

Methodology is described in detail elsewhere [33]. Briefly, leftover clinical samples consisting of EDTA-anticoagulated peripheral blood were diluted in whole blood previously confirmed not to contain any Plasmodium parasites within $48 \mathrm{~h}$ of receipt from the diagnostic laboratory. Pre-dilution parasite densities were determined by expert microscopists counting the number of parasitized cells in a sample size of 10,000 red blood cells on thin blood films to obtain a percentage parasitaemia and converted to parasite density (number of parasites per $\mu \mathrm{L}$ ) using the red cell count. For samples included in the first distribution, a red cell count of $5 \times 10^{12}$ per litre was assumed, while red cell counts in subsequent distributions were determined in the initial, pre-dilution samples using a C-Chip DHC-N01 Disposable haemocytometer (NanoEnTek Inc. via MT Promedt Consulting GmbH, Germany). For cultured parasites, a thin blood film was made from the undiluted culture and a haemocytometer was used from the outset to obtain a red cell count in all cases, in order to obtain the pre-dilution parasite density in parasites per $\mu \mathrm{L}$ of synchronized ring-stage parasites.

For all samples, clinical and cultured, dilution to the desired parasite density was performed using parasitenegative whole blood supplied by UK National Blood and Transplant. To confirm the absence of Plasmodium in the supplied blood used for EQA sample preparation, only blood from seronegative donors was accepted, and blood used for negative samples underwent further PCR confirmation. All positive and negative samples were confirmed by PCR at multiple reference laboratories, again after sample production [5, 9, 34, 35]. Criteria for being a reference laboratory are outlined in the operational manual [33].

The range of parasite densities targeted was: $2 \times 10^{3}$ parasites per microlitre of blood $(\mathrm{p} / \mu \mathrm{L}), 2 \times 10^{2} \mathrm{p} / \mu \mathrm{L}, 2 \times 10^{1}$ $\mathrm{p} / \mu \mathrm{L}, 2 \mathrm{p} / \mu \mathrm{L}, 0.2 \mathrm{p} / \mu \mathrm{L}$, and $0.05 \mathrm{p} / \mu \mathrm{L}$. Aliquots of the dilution series for each sample were then used to prepare dried blood spots (DBS) and/or lyophilized specimens.

To prepare DBS samples, $50 \mu \mathrm{L}$ aliquots were deposited onto Protein Saver 903 cards (GE Healthcare Life Sciences) and air dried for 3-4 h in a tissue culture hood before being packaged in gas-impermeable bags with desiccant [33].

For lyophilization, each whole blood sample was aliquoted in $500 \mu \mathrm{L}$ aliquots and lyophilized using a batch method and tray dryer, in a CHRIST freeze-dryer (EPSILON 2-12DS). Pre-freezing was carried out at $-40{ }^{\circ} \mathrm{C}$ for $3 \mathrm{~h}$. Preparation for sublimation was carried out at $-30{ }^{\circ} \mathrm{C}$ for $30 \mathrm{~min}$. Sublimation was done under the following conditions:

- $\quad-20^{\circ} \mathrm{C}$ for $30 \mathrm{~min}$ at $0.040 \mathrm{mBar}$ pressure

- $\quad-10^{\circ} \mathrm{C}$ for $30 \mathrm{~min}$ at $0.040 \mathrm{mBar}$ pressure

- $10{ }^{\circ} \mathrm{C}$ for $30 \mathrm{~min}$ at $0.030 \mathrm{mBar}$ pressure

- $20^{\circ} \mathrm{C}$ for $2 \mathrm{~h}$ at $0.025 \mathrm{mBar}$ pressure

Secondary drying was carried out for $15 \mathrm{~min}$ at $20^{\circ} \mathrm{C}$ and $0.025 \mathrm{mBar}$ pressure. Vials were stoppered in a vacuum and crimp capped prior to storage and distribution. All samples are kept at $-80^{\circ} \mathrm{C}$ for long-term storage.

\section{Panel composition}

Each panel consisted of five DBS and five lyophilized blood (LB) samples and included both positive and negative samples. Plasmodium falciparum and $P$. vivax samples were sent in all three distributions, while $P$. knowlesi was included in distributions 1 and 2, and Plasmodium malariae in distribution 3 only (Table 1). Samples of these four species had parasite densities ranging from 0.018 to $800 \mathrm{p} / \mu \mathrm{L}$. There were no Plasmodium ovale samples included in distributions 1 to 3 due to a temporary supply shortage, but they have been included in subsequent distributions. Negative samples were included in all distributions in both DBS and LB formats.

\section{Distribution}

Panels of samples were shipped to participating laboratories twice a year. Distribution 1 panel was sent in January 2017, distribution 2 in July 2017 and 
Table 1 Characteristics of external quality assessment panels shipped to participants by distribution

\begin{tabular}{|c|c|c|c|c|}
\hline \multirow[t]{2}{*}{ Distribution } & \multicolumn{2}{|c|}{ Lyophilized blood } & \multicolumn{2}{|c|}{ Dried blood spots } \\
\hline & $\begin{array}{l}\text { Number } \\
\text { of samples }\end{array}$ & $\begin{array}{l}\text { Parasite } \\
\text { density } \\
\text { (parasites/ } \\
\mu \mathrm{L} \text { ) }\end{array}$ & $\begin{array}{l}\text { Number } \\
\text { of samples }\end{array}$ & $\begin{array}{l}\text { Parasite } \\
\text { density } \\
\text { (parasites/ } \\
\mu \mathrm{L} \text { ) }\end{array}$ \\
\hline
\end{tabular}

\begin{tabular}{lllll}
\hline Distribution 1 January 2017 & & & \\
Negative & 2 & - & 2 & - \\
P. falciparum & 0 & - & 3 & $0.05 ; 0.2 ; 2.0$ \\
P. vivax & 2 & $0.018 ; 0.18$ & 0 & - \\
P. knowlesi & 1 & 10 & 0 & - \\
P. malariae & 0 & - & 0 & - \\
Distribution 2-July 2017 & & & \\
Negative & 1 & - & 2 & - \\
P. falciparum & 2 & $0.1 ; 1.0$ & 0 & - \\
P. vivax & 1 & 0.018 & 0 & - \\
P. knowlesi & 1 & 1.0 & 3 & $2.0 ; 2.0 ; 20$ \\
P. malariae & 0 & - & 0 & - \\
Distribution 3-January 2018 & & & \\
Negative & 1 & - & 1 & - \\
P. falciparum & 2 & $20 ; 200$ & 0 & - \\
P. vivax & 1 & 46.5 & 1 & 400 \\
P. knowlesi & 0 & - & 0 & - \\
P. malariae & 1 & 125 & 3 & $125 ; 400 ; 800$ \\
\hline
\end{tabular}

distribution 3 in January 2018. Samples were sent via courier at ambient temperature directly to the majority of laboratories. In some cases they were sent via air freight to the nearest airport for collection.

\section{Reporting}

After shipment of samples, participants were given 8 weeks to submit results via an online portal hosted by UK NEQAS. Participants were asked to use their usual methods for molecular analysis and to report extraction and amplification methods used and the result obtained for each sample. The options on the online portal for the individual samples included identification of each of the five species of human-infecting Plasmodia, the Plasmodium genus, a negative result, or an indeterminate result.

During the three distributions, seven laboratories failed to submit their results to the online portal by the submission deadline but provided results to the coordinators by email shortly thereafter. While these laboratories were not given an official report since they did not meet the deadline, results from these laboratories are included in this paper.

\section{Referee laboratories}

Six referee laboratories were selected based on their technical qualifications, publication record using a range of malaria NAAT methods, available resources to serve as a referee laboratory, and geographic location [33].

\section{Analysis}

Laboratories were asked to submit a 'profile' of their ability to identify positive samples to the genus level and to the species level for each of the five human-infecting Plasmodium species. Interpretation of the results was done by taking into account each site's reported capacity to detect each species in order to ensure that laboratories would not be penalized for identifying false-negatives among samples that they do not have the capacity to detect routinely. In this paper, overall results are presented from all laboratories combined and performance is based on correct or incorrect results according to whether the result submitted matched the real sample, then adjusted according to the laboratory's self-reported capacity to identify each species.

A full reporting scheme is available in the online operational manual [33]. Participants were encouraged to contest their scores, especially if they felt their profiling might have been wrong, but no complaints were received.

Data analysis was performed using Stata 15 (Stata Corp, College Station, USA). Factors which were found to have $\mathrm{p}<0.1$ in the univariate analysis were included in the multivariate model. For determining performance at high and low parasite density, a threshold of 2 $\mathrm{p} / \mu \mathrm{L}$ was selected [36].

\section{Survey}

In September 2018, after participants had experience with four distributions, laboratories were requested to complete an online survey with questions about problems they encountered during their participation in the scheme and if they had used their EQA results to amend the methodology or protocols in place at their site.

\section{Results}

Overall, 57 laboratories had joined the WHO NAAT EQA scheme by distribution 3. Of these, 54 laboratories from 33 countries participated in distributions $1-3$. Not all laboratories participated in all distributions. Participating laboratories included 11 (20\%) from two countries in North America, 10 (19\%) from nine countries in Africa, 12 (22\%) from seven countries in Asia, $12(22 \%)$ from eight countries in South/Central America, eight (15\%) from six countries in Europe, and one (2\%) in Australia. 
Panels were shipped to 55 laboratories in distribution 1, 53 in distribution 2, and 56 in distribution 3. Fortyone laboratories submitted results in distribution 1, 37 in distribution 2, and 45 in distribution 3 (Table 2). More LB samples were analysed than DBS samples in all three distributions. Overall 359, 324 and 394 results were submitted from laboratories in distributions 1,2 , and 3 , respectively.

\section{Laboratory profiles}

Of the 57 laboratories, 51 and 49 reported that they could analyse LB and DBS samples, respectively. All laboratories reported that they could identify $P$. falciparum to the species level. Seven (13\%) laboratories reported that $P$. falciparum was the only species they could identify to species level, while nine (16\%) could identify $P$. falciparum and $P$. vivax only to species level. Twenty-one (38\%) laboratories reported that they could identify all species except $P$. knowlesi, one laboratory (2\%) reported ability to identify all species except $P$. ovale, and 18 (32\%) reported that they could identify all five species. The majority of laboratories reported that they could detect Plasmodium to genus level among species that they could not identify to species level, while some laboratories use species-specific primers, and are therefore unable to detect Plasmodium genus among samples of species that they do not aim to detect. For example, among the 31 laboratories that do not identify P. knowlesi to species level, 19 reported that they could identify Plasmodium among $P$. knowlesi samples.

\section{Methodology used by laboratories}

For nucleic acid extraction, use of a silica column was the most popular method, used in $55.3 \%$ of samples reported (Fig. 1). Chelex was used in $8.6 \%$ of samples, while $20.8 \%$ of samples were analysed using another method.

The most commonly used techniques for nucleic acid amplification were real-time single target PCR and nested PCR, used in 35.3 and $33.2 \%$ of samples analysed, respectively (Fig. 1). Multiplex PCR accounted for $18.5 \%$ of samples, while $2.3 \%$ of samples were analysed using LAMP and other techniques accounted for the remaining $10.7 \%$ of samples analysed. There was no significant difference in amplification technique used by distribution.

\section{Performance}

Based on three panels containing four of the five Plasmodium species infecting humans, across a parasite density range of $0.018-800 \mathrm{p} / \mu \mathrm{L}$, the raw results and the results adjusted taking into consideration laboratory capacity are presented in Fig. 2.

When results were adjusted to take into account the laboratory's reported capacity, the percentage of samples correctly identified as $P$. falciparum was unchanged as all laboratories reported that they had the ability to identify this species. The performance of correct $P$. vivax identification improved slightly to $89.2 \%$ upon adjustment, as only seven laboratories stated they did not have the techniques to identify $P$. vivax. The percentage of $P$. knowlesi samples correctly identified was $69.2 \%, 45.0 \%$ greater than using raw results, as 21 laboratories reported they could not identify P. knowlesi. Some $69.3 \%$ of P. malariae samples were correctly identified using adjusted results.

Based on adjusted results scores there was weak evidence that overall performance improved with each distribution, with $73.3,80.5$ and $83.0 \%$ of samples being correctly identified between distributions 1 and $3(p=0.09)$. The fewest false-positives in negative samples occurred in distribution 2, with $11.9,2.1$ and $10.1 \%$ of false-positives being reported in distributions 1,2 and 3 , respectively $(\mathrm{p}=0.06)$. Detection of $P$. falciparum improved significantly from $43.8 \%$ in distribution 1 to $91.9 \%$ in distribution 3 . Performance against $P$. vivax improved slightly, but not significantly, while neither $P$. knowlesi nor P. malariae samples were in all three distributions and therefore could not be evaluated over time.

Table 2 Characteristics of external quality assessment results submitted by each distribution

\begin{tabular}{|c|c|c|c|}
\hline & Distribution 1 & Distribution 2 & Distribution 3 \\
\hline Panels shipped & 55 & 53 & 56 \\
\hline Participants submitting results ${ }^{a}$ & $41(75 \%)$ & $37(70 \%)$ & $45(80 \%)$ \\
\hline \multicolumn{4}{|c|}{ Number of laboratories which processed } \\
\hline Lyophilized blood & $40(98 \%)$ & $35(95 \%)$ & $43(96 \%)$ \\
\hline Dried blood spots & $32(78 \%)$ & $30(81 \%)$ & $36(80 \%)$ \\
\hline \multicolumn{4}{|l|}{ Number of samples submitted: } \\
\hline Lyophilized blood & 200 & 174 & 214 \\
\hline Dried blood spots & 159 & 150 & 180 \\
\hline Total & 359 & 324 & 394 \\
\hline
\end{tabular}

a Results include samples from laboratories that submitted results after the EQA submission deadline 

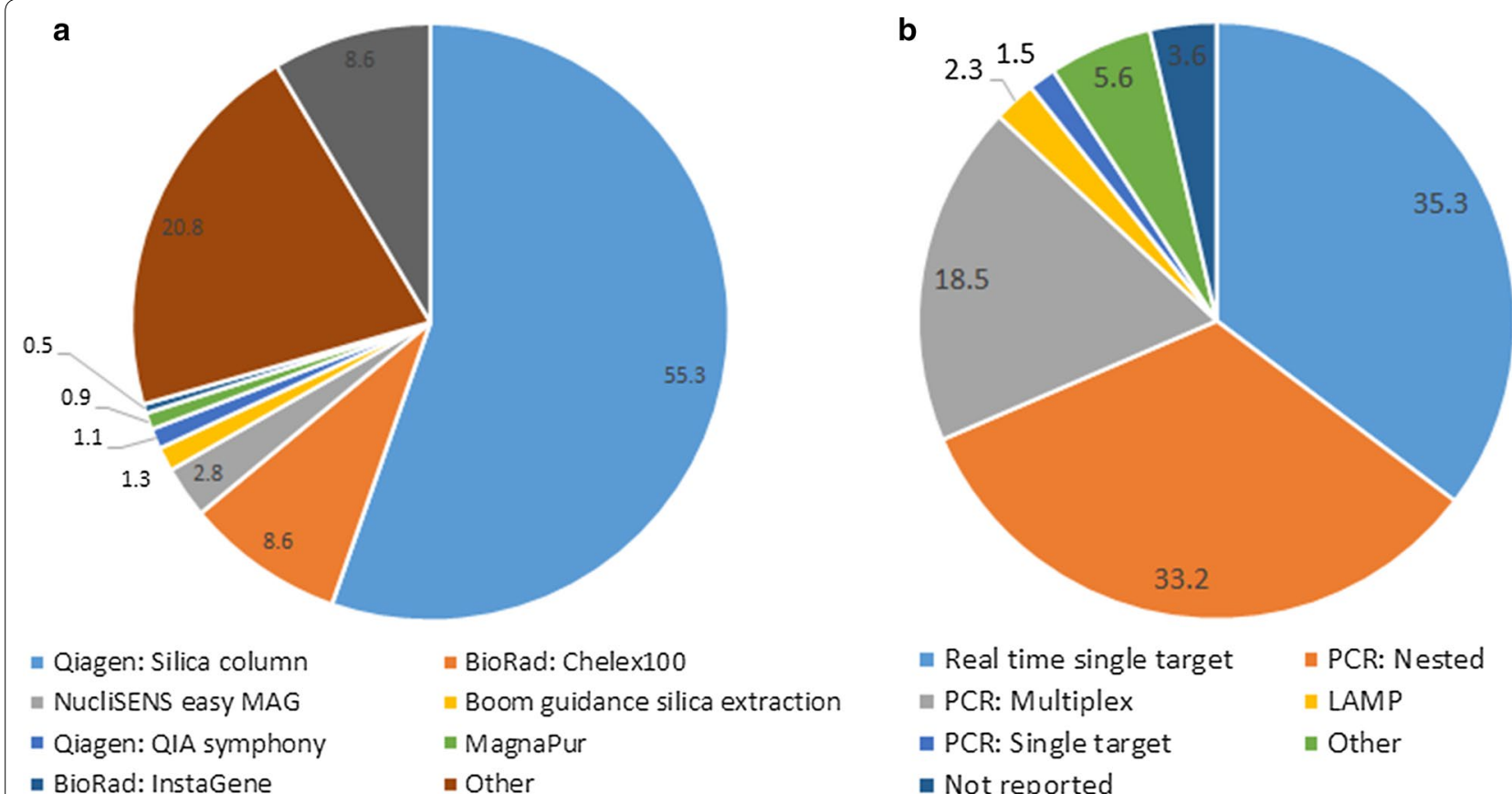

- Not reported

Fig. 1 Characteristics of extraction (a) and amplification (b) methods used by participating laboratories across all three distributions. Percentages of laboratories for each methodology are shown. Methods of extraction and amplification included in other category were not described by participants

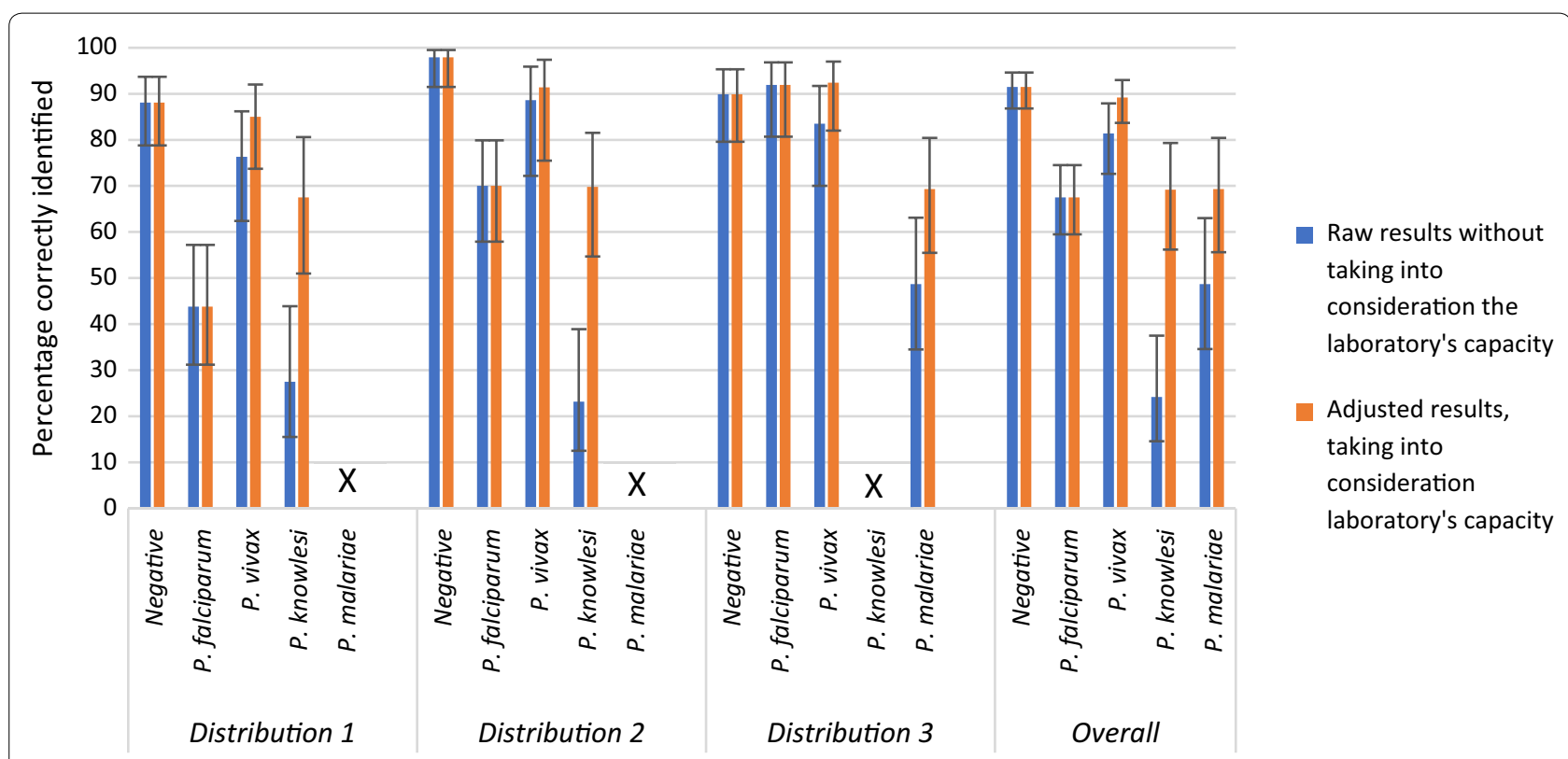

Fig. 2 Accuracy of external quality assessment results by raw and laboratory capacity-adjusted results X: samples of this species were not included in this round

In Fig. 3, results were adjusted to match laboratory capacity for Plasmodium genus detection and species identification. As the concentration of $P$. falciparum decreased, generally the percentage of correctly identified samples also decreased, with $95.4 \%$ of the 200 parasite $/ \mu \mathrm{L}$ samples being correctly identified and $31.3 \%$ of 


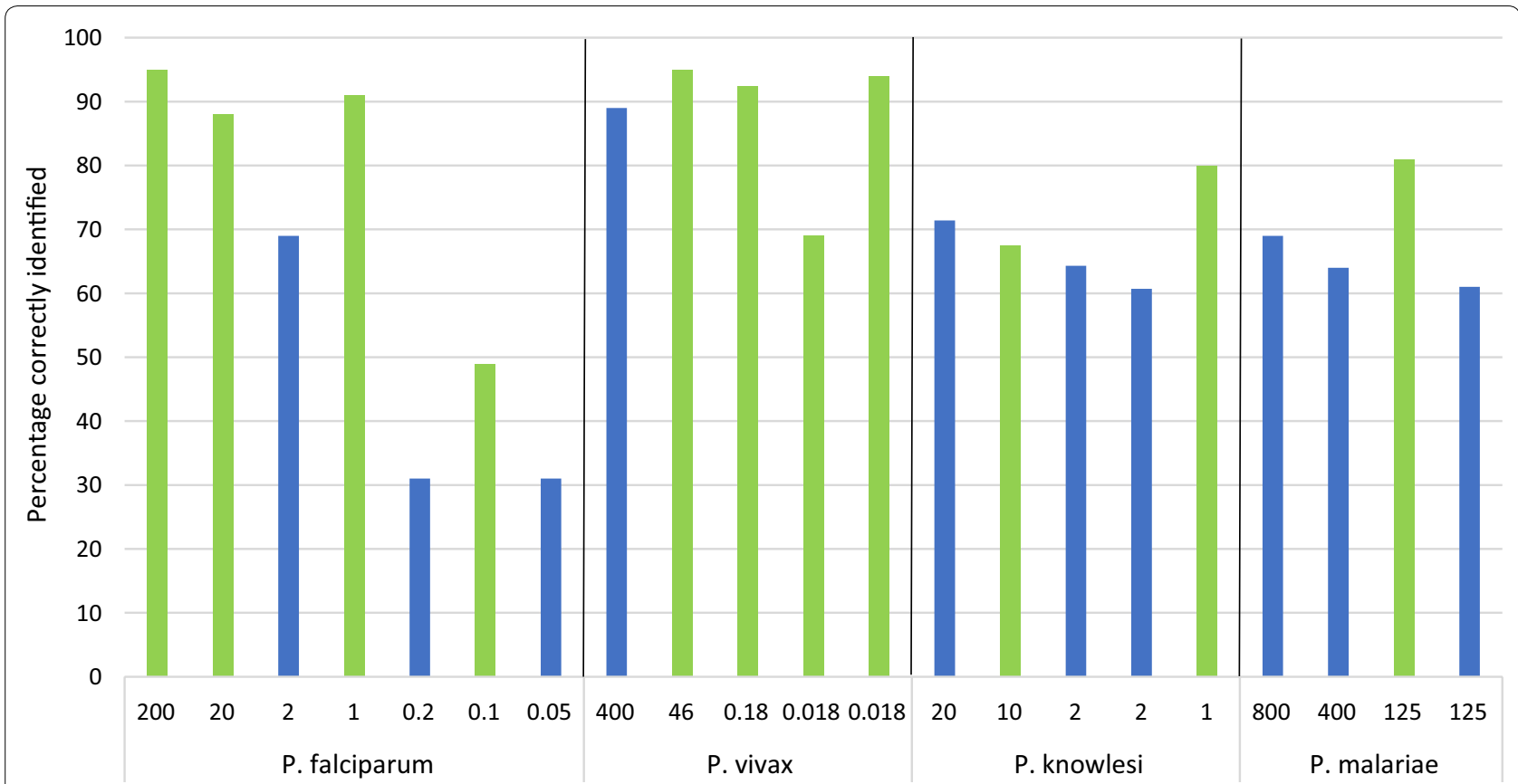

Fig. 3 Accuracy of external quality assessment results on the basis of parasite species and density (parasites/ $\mu \mathrm{L})$. Results adjusted for laboratory capacity. Green bars denote lyophilized blood samples; blue bars denote dried blood spots

Table 3 Accuracy of external quality assessment results above and below a density of 2 parasites/ $\mu \mathrm{L}$

\begin{tabular}{|c|c|c|c|}
\hline & \multicolumn{2}{|c|}{$\begin{array}{l}\text { Percentage of samples correctly } \\
\text { identified }^{\mathrm{a}}\end{array}$} & \multirow[t]{2}{*}{$P$ value } \\
\hline & $\begin{array}{l}\leq 2 \text { parasites/ } \\
\mu \mathrm{L}\end{array}$ & $>2$ parasites $/ \mu \mathrm{L}$ & \\
\hline \multicolumn{4}{|l|}{ Species } \\
\hline P. falciparum & 54.8 & 91.9 & $<0.01$ \\
\hline P. vivax & 86.9 & 92.5 & 0.32 \\
\hline P. knowlesi & 69.2 & 69.1 & 0.98 \\
\hline P. malaria ${ }^{b}$ & NA & 48.7 & NA \\
\hline \multicolumn{4}{|l|}{ Sample type } \\
\hline Dried blood spots & 50.1 & 70.9 & 0.02 \\
\hline Lyophilized blood & 80.5 & 85.8 & 0.18 \\
\hline Overall & 68.3 & 79.1 & 0.03 \\
\hline
\end{tabular}

a Results adjusted for laboratory capacity

b All samples of P. malariae were $>2$ parasites $/ \mu \mathrm{L}$

the sample at 0.05 parasites $/ \mu \mathrm{L}$ being identified $(\mathrm{p}<0.01)$. This trend was not seen for any of the other three species.

When applying a target NAAT detection threshold of 2 parasites/ $\mu \mathrm{L}$, adjusted performance was $79.1 \%$ against samples $>2$ parasites $/ \mu \mathrm{L}$ and $68.3 \%$ for samples $\leq 2$ parasites $/ \mu \mathrm{L}(\mathrm{p}=0.03)$ (Table 3$)$. This was driven by the difference in performance against $P$. falciparum at different densities, with $91.7 \%$ of $P$. falciparum samples $>2$ parasites $/ \mu \mathrm{L}$ identified correctly and $54.8 \%$ of samples below this density $(\mathrm{p}<0.01)$. There was no significant difference for $P$. vivax or $P$. knowlesi, and all samples of P. malariae were $>2$ parasites $/ \mu \mathrm{L}$ so a comparison could not be made. Performance against the higher concentration of DBS samples was 20.8 percentage points higher than the lower concentration; however, there was no significant difference against the LB samples.

Participating laboratories detected lyophilized samples better than DBS samples, with 85.4 and $70.0 \%$ of samples, respectively, being identified correctly $(\mathrm{p}<0.01)$ (Table 4). While performance was better than with DBS samples in all three distributions, the difference was only significant in distributions 1 and 3 $(\mathrm{p}<0.01)$ and not in distribution $2(\mathrm{p}=0.5)$.

Table 4 illustrates factors found significantly to affect adjusted performance, including parasite density, type of sample, species, and being a reference laboratory. The region in which the laboratory was located, type of nucleic acid amplified, amplification method, and extraction method did not significantly impact performance.

Overall adjusted performance varied between 20 and $100 \%$ across participating laboratories, with 46 (85\%) laboratories correctly identifying $>70 \%$ of the samples that they tested. Twenty-six laboratories participated in all three distributions. Of these, seven laboratories (27\%) scored $80 \%$ or higher in all three distributions, while 
Table 4 Result outcomes in relation to laboratory, test and sample characteristics

\begin{tabular}{|c|c|c|c|c|c|c|c|}
\hline Characteristic & $\begin{array}{l}\text { Number } \\
\text { of samples } \\
\text { analysed }\end{array}$ & $\begin{array}{l}\% \text { Correct based on results } \\
\text { adjusted for laboratory } \\
\text { capacity }\end{array}$ & $P$ & Odds ratio unadjusted* & $P$ & Odds ratio adjusted ${ }^{a}$ & $P$ \\
\hline \multicolumn{8}{|l|}{ Region } \\
\hline Africa & 175 & $77.5(69.4-84.0)$ & \multirow[t]{6}{*}{0.93} & 1 & \multirow[t]{6}{*}{0.73} & & \\
\hline Asia & 250 & $78.8(70.8-85.1)$ & & $1.1(0.7-1.7)$ & & & \\
\hline Europe & 130 & $79.2(68.6-87.0)$ & & $1.1(0.6-1.9)$ & & & \\
\hline North America & 174 & $75.9(64.9-84.2)$ & & $0.9(0.6-1.5)$ & & & \\
\hline South/Central America & 319 & $79.3(74.4-83.5)$ & & $1.1(0.7-1.7)$ & & & \\
\hline Oceania & 29 & $82.8(82.8-82.8)$ & & $1.4(0.5-3.9)$ & & & \\
\hline \multicolumn{8}{|l|}{ Reference laboratory } \\
\hline Yes & 114 & $83.3(79.3-86.7)$ & \multirow[t]{2}{*}{0.06} & 1 & \multirow[t]{2}{*}{0.07} & 1 & \multirow[t]{2}{*}{0.02} \\
\hline No & 963 & 78.5 (75.8-81.8) & & $0.7(0.5-1.0)$ & & $0.7(0.5-0.9)$ & \\
\hline \multicolumn{8}{|l|}{ Type of sample } \\
\hline Dried blood spots & 489 & $70.0(65.5-74.1)$ & \multirow[t]{2}{*}{$<0.01$} & 1 & \multirow[t]{2}{*}{$<0.01$} & 1 & \multirow[t]{2}{*}{$<0.01$} \\
\hline Lyophilized & 588 & $85.4(81.4-88.6)$ & & $2.5(1.9-3.4)$ & & $2.3(1.7-3.2)$ & \\
\hline \multicolumn{8}{|l|}{ Species } \\
\hline Negative & 316 & $91.5(86.8-95.6)$ & \multirow[t]{5}{*}{$<0.01$} & 1 & \multirow[t]{5}{*}{$<0.01$} & 1 & \multirow[t]{5}{*}{$<0.01$} \\
\hline P. falciparum & 252 & $67.5(59.5-74.5)$ & & $0.2(0.2-0.4)$ & & $0.5(0.2-1.4)$ & \\
\hline P. vivax & 194 & $89.2(83.7-93.0)$ & & $0.8(0.4-1.5)$ & & $2.6(0.8-8.9)$ & \\
\hline P. knowlesi & 165 & $69.2(56.9-79.3)$ & & $0.2(0.2-0.4)$ & & $0.3(0.1-0.8)$ & \\
\hline P. malariae & 150 & $69.3(55.6-80.4)$ & & $0.2(0.1-0.4)$ & & $0.2(0.1-0.5)$ & \\
\hline \multicolumn{8}{|c|}{ Plasmodium nucleic acid present } \\
\hline Yes & 761 & $73.8(75.1-81.4)$ & \multirow[t]{2}{*}{$<0.01$} & 1 & \multirow[t]{2}{*}{$<0.01$} & - & \\
\hline No & 316 & $91.5(86.8-94.6)$ & & $3.8(2.5-5.8)$ & & & \\
\hline \multicolumn{8}{|l|}{ Parasite density (parasites $/ \mu \mathrm{L}$ ) } \\
\hline Negative & 316 & $91.5(86.8-94.6)$ & \multirow[t]{5}{*}{$<0.01$} & 1 & 0.01 & 1 & 0.03 \\
\hline$>0-<1$ & 14 & $64.0(56.0-71.4)$ & & $0.2(0.1-0.3)$ & & $0.1(0.04-0.4)$ & \\
\hline$\geq 1-<10$ & 162 & $74.1(63.9-82.1)$ & & $0.3(0.2-0.5)$ & & $0.7(0.3-2.0)$ & \\
\hline$\geq 10-<100$ & 156 & $81.8(74.4-87.4)$ & & $0.40 .2-0.7)$ & & $0.7(0.3-1.5)$ & \\
\hline$\geq 100$ & 229 & $77.3(84.2-93.3)$ & & $0.3(0.2-0.5)$ & & - & \\
\hline Extraction method & & & & & & & \\
\hline Qiagen: Silica column & 596 & $79.9(76.0-.83 .3)$ & 0.62 & 1 & 0.17 & & \\
\hline BioRad: Chelex100 & 90 & $76.7(64.0-85.9)$ & & $0.8(0.5-1.4)$ & & & \\
\hline NucliSENS easy MAG & 30 & $90.0(84.1-93.9)$ & & $2.3(0.6-7.6)$ & & & \\
\hline $\begin{array}{l}\text { Boom guidance silica extrac- } \\
\text { tion }\end{array}$ & 14 & $92.9(92.9-92.9)$ & & $3.2(0.4-25.3)$ & & & \\
\hline Qiagen: QIA symphony & 12 & 100 & & - & & & \\
\hline MagnaPur & 10 & 100 & & - & & & \\
\hline BioRad: InstaGene & 5 & $60.0(60.0-60.0)$ & & $0.4(0.1-2.3)$ & & & \\
\hline Other & 224 & $73.7(65.7-80.3)$ & & $0.7(0.5-1.0)$ & & & \\
\hline Not reported & 90 & $79.0(59.4-90.5)$ & & $0.9(0.5-1.6)$ & & & \\
\hline Amplification method & & & & & & & \\
\hline Real time single target & 380 & $81.8(76.3-86.3)$ & 0.50 & 1 & 0.97 & & \\
\hline Nested PCR & 358 & $75.4(68.6-81.2)$ & & $0.7(0.5-1.0)$ & & & \\
\hline Multiplex PCR & 199 & $79.4(71.2-85.7)$ & & $0.9(0.6-1.3)$ & & & \\
\hline LAMP & 19 & $68.4(41.2-87.0)$ & & $0.5(0.2-1.3)$ & & & \\
\hline Single target $P C R$ & 16 & $75.0(70.3-79.2)$ & & $0.7(0.2-2.1)$ & & & \\
\hline Other & 60 & $85.0(67.8-93.9)$ & & $1.3(0.6-2.7)$ & & & \\
\hline Not reported & 39 & $79.5(57.6-91.7)$ & & $0.9(0.4-2.0)$ & & & \\
\hline Type of nucleic acid & & & & & & & \\
\hline DNA & 995 & $77.8(74.3-80.9)$ & 0.47 & 1 & 0.29 & & \\
\hline RNA & 45 & $84.4(62.1-94.7)$ & & $1.6(0.7-3.5)$ & & & \\
\hline
\end{tabular}

a Odds ratios (unadjusted and adjusted) show the ratio for each line compared to the first line in the section 
19 (73\%) laboratories scored $60 \%$ or higher in all three distributions.

\section{Challenges for EQA participants and the provider}

Most laboratories $(85 \%, 46 / 54)$ surveyed provided information about challenges they faced during their participation in the scheme. The main challenge related to delays in obtaining samples, mainly due to customs requirements, often compounded by the airport collection point being far from the laboratory. Out of the laboratories that completed the survey, nine $(20 \%)$ reported consistently having problems with shipment delays while five laboratories (11\%) reported having problems in only some of the distributions. Problems with entering data into the submission portal were reported. Other challenges included not having any or sufficient quantities of reagents or laboratory supplies to analyse the samples before the deadline and not having personnel available for various reasons (e.g., staff in the field).

From an EQA provision perspective there were numerous challenges, mostly related to identifying the correct contact person for each laboratory, issues related to import permits, and identifying relevant courier partners for shipment to certain geographic regions. Language barriers and working across time zones added to the complexity. The reporting time allowed was extended in the first distribution in order to accommodate these difficulties.

\section{How laboratories have used their EQA results}

Of 46 laboratories that provided information about their participation, 13 (31\%) responded that they changed or amended their laboratory protocol(s) in response to their results from the EQA scheme. Of these laboratories, changing the protocol of the extraction and/or amplification processes were the main responses (four laboratories), while two other laboratories reported adding new PCR methods to their existing methods. Three laboratories reported that the scheme allowed them to identify additional species that they previously did not identify by testing and refining their protocols for these new species during the scheme. Two laboratories reported adding $P$. knowlesi identification and one other laboratory $P$. knowlesi, P. malariae and P. ovale.

\section{Discussion}

The use of NAATs for malaria diagnosis in research and clinical care has increased dramatically over the last two decades. Clinical trials of new anti-malarial drugs or vaccines require precise, reliable methods for parasite detection, and low density of parasitaemia may be missed by routine diagnostic methods, such as microscopy and RDTs. NAAT can also be used for high throughput of samples which is not possible using other methods. The continual evolution of NAATs and the lack of standardized methods highlight the need for an EQA scheme to ensure safe laboratory performance and, over the longer term, potentially to facilitate assay harmonization. The scheme is also a mechanism to promote use of and reporting based on the available International DNA Standard and to advocate for the creation of more such standards (e.g., the need for an International Standard for RNA-based assays).

Upon recommendations of $\mathrm{WHO}$ and building on the experiences of UK NEQAS and others in the research community $[16,31,37]$, this new international scheme is unique in its geographic coverage, panel sample format and composition and breadth of participation. The scheme thus allows laboratories to compare their performance with their global peers, across various specimen types, including all five malaria species and, over a suitable time period, to assess the effect of changes that might have been introduced as a direct effect of participation within this EQA scheme. Although data from more distributions will be needed to draw broader conclusions and assess the impact, this analysis already presents some interesting findings and challenges.

Firstly, false-positives against malaria-negative samples were less common than false-negatives against Plasmodium-containing samples. The false-positive rate was $8.5 \%$ compared to a $26.2 \%$ false-negative rate. The higher false-negative rate likely reflects the lower parasite densities of distributed $P$. falciparum samples since higher density samples $>2$ parasites $/ \mu \mathrm{L}$ were more reliably detected (e.g., 7.5\% false-negative rate for such $P$. vivax samples and $8.1 \%$ false-negative rate for such $P$. falciparum samples). These data show that misalignment in laboratory results can be expected, especially as the parasite density goes down.

The scheme included DBS because this is the most common sample format used in research in endemic countries due to its low cost, ease of transport and storage, and stability [38]. Performance was significantly higher against $500 \mu \mathrm{L}$ LB samples than $50 \mu \mathrm{L}$ DBS, especially at concentrations below $2 \mathrm{p} / \mu \mathrm{L}$. This is consistent with reported findings [39] and needs to be taken into consideration when comparing results of studies using different sample types. Inclusion of paired samples in future distributions will allow for a more direct comparison between performance of DBS and LB samples. Laboratories were not asked to provide references or specific protocol details, such as how much of the LB and/ or DBS sample was tested in their protocol. Requesting this information could allow the effect of sample quantity used on performance to be assessed. 
All of the factors that were found to significantly affect performance were due to the characteristics of the samples themselves (format, species, parasite density), and not the laboratory location (geography) or the reported methodology used (nucleic acid extraction, amplification, or DNA vs RNA target) with the exception that referee laboratories performed better than non-referee laboratories. This would suggest that referee laboratories could play an important role in shaping malaria molecular testing practice through publication of protocols and mentoring.

To overcome the challenges identified by participants, some revisions were implemented in the EQA scheme, including a requirement to supply copies of import permits prior to shipment, translation of key documents into host country language and, in some cases, centralized shipment to a WHO country office for subsequent onward shipment to participating laboratories. The turnaround time for reporting results was extended from 6-8 weeks. Participation was also restricted to those laboratories regularly conducting NAATs to avoid failure to submit results caused by lack of reagents, lack of personnel, or lack of ongoing studies to support testing. The online data entry interface was adapted based on feedback to improve user-friendliness. In response to challenges brought to the attention of the EQA provider, various changes were incorporated, including creating a separate inbox for query handling, sending reminder emails to participants for import permits and other relevant documentation, including a list of laboratory profiles toward the end of EQA reports for each distribution, to illustrate the rationale for scoring and rewording the information section of the participant portal better to explain the data entry procedure.

As a result of their involvement in the scheme, several laboratories amended their protocols or included new methods, with some laboratories including new species into the range tested. One important aim of the scheme was to enable laboratories to learn from their results and from each other on ways to improve performance.

Certain limitations of the study must be acknowledged. As not all laboratories participated in all distributions, and due to the different panel composition during each distribution, both in terms of species and parasite density, it is not possible to compare performance directly over time with only three distributions. As the EQA scheme progresses and more data are collected, it will be interesting to assess whether there are more significant changes in performance over time, best demonstrated by trends in individual laboratories. Samples were diluted and parasite densities determined by UK NEQAS. Calibration of EQA materials with the International DNA Standard could also be performed and is done in one of the referee laboratories. However, all International Standards are expressed in International Units, whereas clinical laboratories and WHO work in parasites/volume or copies/ volume. The EQA programme chose a density threshold of $2 \mathrm{p} / \mu \mathrm{L}$ to determine 'adequate' performance, the sensitivity of a test required to detect asymptomatic infections and those in low-density populations [36]. The sensitivity required of a test can depend on the purpose of testing, with target sensitivity for drug and vaccine efficacy trials being greater than that required for case management, so perhaps one threshold for a given assay is not sufficient as an indicator of its utility. For instance, the limit of detection for assays intended to support CHMI studies is generally lower $(0.01-0.05$ parasites $/ \mu \mathrm{L})$. However, whether the scheme uses one or more thresholds, the critical point is that individual laboratories may use this scheme to obtain objective performance data, including the limit of detection for the test(s) they use and undertake quality improvement by investigating any failures. These will all help them select assays appropriate for the specific samples they are asked to examine.

This scheme intends to evolve in the constantly changing malaria landscape. For example, future distributions of the scheme will include $p f h r p 2-$ and $p f h r p 3$-negative $P$. falciparum samples, allowing laboratories that analyse these genes to assess their accuracy at identifying samples with gene deletions. It is critical that surveys for pfhrp $2 / 3$ gene deletions should yield accurate and reliable results, as over- or under-calling their presence will have a significant impact on policy decisions on the most appropriate malaria RDTs to be used in these areas.

While the scheme is currently free for all participating laboratories, this arrangement is unsustainable in the long term. In the near future, user fees will be introduced to cover costs, including the replenishment of EQA materials. Costs are expected to be between US\$300-350 per laboratory per distribution, which is an extremely small fraction of the cost of clinical and epidemiological research budgets, and a small investment to safeguard against decision-making based on flawed data. Donors funding malaria research and laboratory capacity strengthening should require laboratories to participate in EQA schemes, as is a requirement for reference laboratories in countries with mature quality assurance systems. For laboratories that do not originate from low or middle income countries (LMICs) or participate in both the UK NEQAS and WHO schemes, participation in the UK NEQAS Malaria molecular scheme will be encouraged so that more laboratories from LMICs can participate in the WHO scheme. Thus, ongoing EQA for malaria NAATs can help to safeguard clinical trial, clinical care (in some high-resource settings), and research activities that rely on these increasingly important tests. 


\section{Conclusions}

William Osler is quoted as saying "As is our pathology, so is our practice" [40]. As the range and sophistication of diagnostic tests has increased, so has the relevance of his comment. Furthermore, at long last, reliable, qualityassured diagnostic tests for malaria are available for use in resource-poor settings. As technology advances yet further, that repertoire will increasingly include NAAT. But to deploy them without ensuring good performance on the ground would be a major error that could adversely influence decisions affecting both individual patients and whole populations.

\section{Abbreviations \\ CHMI: Controlled human malaria infection; DBS: Dried blood spot; DNA: Deoxyribonucleic acid; EQA: External quality assessment; LAMP: Loop-medi- ated isothermal amplifications; LB: Lyophilized blood; LSHTM: London School of Hygiene and Tropical Medicine; NAAT: Nucleic acid amplification technique; PCR: Polymerase chain reaction; RDT: Rapid diagnostic test; RNA: Ribonucleic acid; WHO: World Health Organization.}

\section{Acknowledgements}

The authors would like the thank the participants of the EQA scheme for the use of the results from their submissions in this manuscript. Thank you to the UK NEQAS Microbiology team for assistance with the EQA provision; NEQAS (Birmingham Quality) for IT support; the participating laboratories and those that served as referees for pre-distribution quality checks of all specimens; and Dr Don van Schalkwyk from the LSHTM for malaria parasite cultures. Thank you to Colin Sutherland from LSHTM and Nick Edwards from the University of Oxford for their input.

\section{Authors' contributions}

JC, PC and JS designed and coordinated the study. RT analysed the data. RT, JC and SM drafted the original manuscript. AS conducted laboratory work including EQA sample preparation. SM, MA, XD, SI, EL, NL, DM and SN provided expert advice on developing the EQA scheme. All authors read and approved the final manuscript.

\section{Funding}

This work was supported using funds from the Department of Foreign Affairs and Trade, Australian Government.

\section{Availability of data and materials}

The datasets used and analysed during the current study are available from the corresponding author on reasonable request.

\section{Ethics approval and consent to participate}

Samples for EQA material were prepared from leftover clinical specimens from patients attending the Hospital for Tropical Diseases in London and from residual blood samples referred from other hospitals across the United Kingdom (UK). Under the terms of the UK Human Tissue Act, 2004, Schedule 4, Section 45, supplementary part 2, ethical approval to use such samples for EQA purposes is not required.

\section{Consent for publication}

Not applicable.

\section{Competing interests}

The authors declare that they have no competing interests.

\section{Author details}

${ }^{1}$ World Health Organization, Geneva, Switzerland. ${ }^{2}$ Independent Consultant, London, UK. ${ }^{3}$ Seattle Malaria Clinical Trials Center, Fred Hutchinson Cancer Research Center, Seattle, USA. ${ }^{4}$ Department of Communicable Diseases and Health Analysis, Pan American Health Organization/World Health
Organization, Washington, DC, USA. ${ }^{5}$ Foundation for Innovative New Diagnostics, Geneva, Switzerland. ${ }^{6}$ Biology of Host-Parasite Interactions Unit, Institut Pasteur, INSERM U1201/CNRS ERL9195, Paris, France. ${ }^{7}$ Malaria Branch, Division of Parasitic Diseases and MalariaCenter for Global Health, Centers for Disease Control and Prevention, Atlanta, USA. ${ }^{8}$ Department of Pathology, School of Biomedical Science, Makerere University, Kampala, Uganda. ${ }^{9}$ UK NEQAS Parasitology, Public Health England, London, UK. ${ }^{10}$ The Hospital for Tropical Diseases, London, UK.

Received: 13 August 2019 Accepted: 23 March 2020

Published online: 30 March 2020

\section{References}

1. WHO. World Malaria Report 2018. Geneva, World Health Organization, 2018.

2. WHO. Guidelines for the treatment of malaria. 2nd Edn. Geneva, World Health Organization, 2010.

3. Waters AP, McCutchan TF. Rapid, sensitive diagnosis of malaria based on ribosomal RNA. Lancet. 1989;1:1343-6.

4. Snounou G, Viriyakosol S, Jarra W, Thaithong S, Brown KN. Identification of the four human malaria parasite species in field samples by the polymerase chain reaction and detection of a high prevalence of mixed infections. Mol Biochem Parasitol. 1993;58:283-92.

5. Snounou G, Viriyakosol S, Zhu XP, Jarra W, Pinheiro L, do Rosario VE, et al. High sensitivity of detection of human malaria parasites by the use of nested polymerase chain reaction. Mol Biochem Parasitol. 1993;61:315-20.

6. Rougemont M, Van Saanen M, Sahli R, Hinrikson HP, Bille J, Jaton K. Detection of four Plasmodium species in blood from humans by $18 \mathrm{~S}$ rRNA gene subunit-based and species-specific real-time PCR assays. J Clin Microbiol. 2004:42:5636-43.

7. Perandin F, Manca N, Calderaro A, Piccolo G, Galati L, Ricci L, et al. Development of a real-time PCR assay for detection of Plasmodium falciparum, Plasmodium vivax, and Plasmodium ovale for routine clinical diagnosis. J Clin Microbiol. 2004:42:1214-9.

8. Rubio JM, Benito A, Berzosa PJ, Roche J, Puente S, Subirats M, et al. Usefulness of seminested multiplex PCR in surveillance of imported malaria in Spain. J Clin Microbiol. 1999:37:3260-4.

9. Murphy SC, Prentice JL, Williamson K, Wallis CK, Fang FC, Fried M, et al. Real-time quantitative reverse transcription PCR for monitoring of bloodstage Plasmodium falciparum infections in malaria human challenge trials. Am J Trop Med Hyg. 2012;86:383-94.

10. Poon LL, Wong BW, Ma EH, Chan KH, Chow LM, Abeyewickreme W, et al. Sensitive and inexpensive molecular test for falciparum malaria: detecting Plasmodium falciparum DNA directly from heat-treated blood by loop-mediated isothermal amplification. Clin Chem. 2006:52:303-6.

11. Han ET, Watanabe R, Sattabongkot J, Khuntirat B, Sirichaisinthop J, Iriko $\mathrm{H}$, et al. Detection of four Plasmodium species by genus- and speciesspecific loop-mediated isothermal amplification for clinical diagnosis. J Clin Microbiol. 2007;45:2521-8.

12. Imwong M, Hanchana S, Malleret B, Renia L, Day NP, Dondorp A, et al High-throughput ultrasensitive molecular techniques for quantifying low-density malaria parasitemias. J Clin Microbiol. 2014:52:3303-9.

13. Hodgson SH, Douglas AD, Edwards NJ, Kimani D, Elias SC, Chang M, et al. Increased sample volume and use of quantitative reverse-transcription PCR can improve prediction of liver-to-blood inoculum size in controlled human malaria infection studies. Malar J. 2015;14:33.

14. Murphy SC, Shott JP, Parikh S, Etter P, Prescott WR, Stewart VA. Malaria diagnostics in clinical trials. Am J Trop Med Hyg. 2013;89:824-39.

15. Hanscheid T, Grobusch MP. How useful is PCR in the diagnosis of malaria? Trends Parasitol. 2002;18:395-8.

16. Murphy SC, Hermsen CC, Douglas AD, Edwards NJ, Petersen I, Fahle GA, et al. External quality assurance of malaria nucleic acid testing for clinical trials and eradication surveillance. PLoS ONE. 2014:9:e97398.

17. Okell LC, Ghani AC, Lyons E, Drakeley CJ. Submicroscopic infection in Plasmodium falciparum-endemic populations: a systematic review and meta-analysis. J Infect Dis. 2009;200:1509-17. 
18. Cheng Q, Cunningham J, Gatton ML. Systematic review of sub-microscopic P. vivax infections: prevalence and determining factors. PLoS Negl Trop Dis. 2015:9:3413.

19. Schneider P, Bousema JT, Gouagna LC, Otieno S, van de Vegte-Bolmer M, Omar SA, et al. Submicroscopic Plasmodium falciparum gametocyte densities frequently result in mosquito infection. Am J Trop Med Hyg. 2007;76:470-4.

20. Tadesse FG, Slater HC, Chali W, Teelen K, Lanke K, Belachew M, et al. The relative contribution of symptomatic and asymptomatic Plasmodium vivax and Plasmodium falciparum infections to the infectious reservoir in a low-endemic setting in Ethiopia. Clin Infect Dis. 2018;66:1883-91.

21. Landier J, Parker DM, Thu AM, Lwin KM, Delmas G, Nosten FH, et al. Effect of generalised access to early diagnosis and treatment and targeted mass drug administration on Plasmodium falciparum malaria in Eastern Myanmar: an observational study of a regional elimination programme. Lancet. 2018;391:1916-26.

22. Wallace PS, MacKay WG. Quality in the molecular microbiology laboratory. Methods Mol Biol. 2012;943:49-79.

23. Woods Tim. Caring, Concern and Consistency. Pathologist. 2018;518:28-31.

24. Jones GR. The role of EQA in harmonization in laboratory medicine-a global effort. Biochem Med. 2017;27:23-9.

25. Shrivastava J. Assessors assemble: the need for harmonised external quality assessment schemes for emerging diagnostic methodologies in the field of parasitology. Trans R Soc Trop Med Hyg. 2019;113:820-2.

26. Alemayehu S, Feghali KC, Cowden J, Komisar J, Ockenhouse CF, Kamau E. Comparative evaluation of published real-time PCR assays for the detection of malaria following MIQE guidelines. Malar J. 2013;12:277.

27. Bustin SA, Benes V, Garson JA, Hellemans J, Huggett J, Kubista M, et al. The MIQE guidelines: minimum information for publication of quantitative real-time PCR experiments. Clin Chem. 2009;55:611-22.

28. Padley DJ, Heath AB, Sutherland C, Chiodini PL, Baylis SA, Collaborative Study Group. Establishment of the 1st World Health Organization International Standard for Plasmodium falciparum DNA for nucleic acid amplification technique (NAT)-based assays. Malar J. 2008;7:139.

29. Kettelhut MM, Chiodini PL, Edwards H, Moody A. External quality assessment schemes raise standards: evidence from the UKNEQAS parasitology subschemes. J Clin Pathol. 2003;56:927-32.
30. WHO. Malaria rapid diagnostic test performance: Results of WHO product testing of malaria RDTs: round 8 (2016-2018). Geneva, World Health Organization, 2018.

31. WHO. WHO external quality assurance scheme for malaria nucleic acid amplification testing. London. Geneva: UK. Meeting report; 2015. p. 2015.

32. Human Tissue Act 2004, United Kingdom. https://www.legislation.gov. uk/ukpga/2004/30/contents.

33. WHO. WHO external quality assurance scheme for malaria nucleic acid amplification testing. Operational Manual. Geneva, World Health Organization, 2018.

34. Canier L, Khim N, Kim S, Sluydts V, Heng S, Dourng D, et al. An innovative tool for moving malaria PCR detection of parasite reservoir into the field. Malar J. 2013;12:405.

35. Rockett RJ, Tozer SJ, Peatey C, Bialasiewicz S, Whiley DM, Nissen MD, et al. A real-time, quantitative $P C R$ method using hydrolysis probes for the monitoring of Plasmodium falciparum load in experimentally infected human volunteers. Malar J. 2011;10:48.

36. WHO. Policy brief on malaria diagnostics in low-transmission settings. Geneva, World Health Organization, 2014.

37. United Kingdom National External Quality Assurance Scheme (UK NEQAS). https://ukneqas.org.uk/.

38. Singh B, Cox-Singh J, Miller AO, Abdullah MS, Snounou G, Rahman HA. Detection of malaria in Malaysia by nested polymerase chain reaction amplification of dried blood spots on filter papers. Trans R Soc Trop Med Hyg. 1996;90:519-21.

39. Strom GE, Tellevik MG, Hanevik K, Langeland N, Blomberg B. Comparison of four methods for extracting DNA from dried blood on filter paper for PCR targeting the mitochondrial Plasmodium genome. Trans R Soc Trop Med Hyg. 2014;108:488-94.

40. Wright JR Jr. Osler's "quote": "as is our pathology so is our practice". Pathol Res Pract. 2013;209:264-5.

\section{Publisher's Note}

Springer Nature remains neutral with regard to jurisdictional claims in published maps and institutional affiliations.
Ready to submit your research? Choose BMC and benefit from:

- fast, convenient online submission

- thorough peer review by experienced researchers in your field

- rapid publication on acceptance

- support for research data, including large and complex data types

- gold Open Access which fosters wider collaboration and increased citations

- maximum visibility for your research: over 100M website views per year

At BMC, research is always in progress.

Learn more biomedcentral.com/submissions 